\title{
Correction to: Scored minor criteria for severe community-acquired pneumonia predicted better
}

\author{
Qi Guo ${ }^{1,2^{*}}$, Wei-dong Song ${ }^{1 \dagger}$, Hai-yan Li ${ }^{3+}$, Yi-ping Zhou ${ }^{2}$, Ming Li ${ }^{2}$, Xiao-ke Chen², Hui Liu ${ }^{2}$, Hong-lin Peng ${ }^{2}$, \\ Hai-qiong Yư ${ }^{2}$, Xia Chen², Nian Liư ${ }^{2}$, Zhong-dong Lü', Li-hua Liang ${ }^{4}$, Qing-zhou Zhao ${ }^{4}$ and Mei Jiang ${ }^{5}$
}

\author{
Correction to: Respir Res (2019) 20:22 \\ https://doi.org/10.1186/s12931-019-0991-4
}

Although the focus of our article in Respiratory Research [1] reports some novel data and has a different focus compared to our publications in The American Journal of the Medical Sciences [2] and Respiratory Medicine [3], we acknowledge that we have duplicated some text and used the same study populations within this article [1] as our previous articles [2, 3].

The database used for the article published in Respiratory Research [1] was the basic database, which came to the conclusion that the individual 2007 IDSA/ATS minor criteria for severe community-acquired pneumonia (CAP) were of unequal weight in predicting hospital mortality, SOFA scores, hospital length of stay, and costs. The retrospective database used for the article published in The American Journal of the Medical Sciences [2] was the same in Respiratory Medicine [3]. The databases used for the article published in Respiratory Research [1] were the same as those in The American Journal of the Medical Sciences [2]. The two articles were based on the same basic theory that 2007 IDSA/ ATS minor criteria for severe CAP were of unequal weight in prediction. We introduced some interesting findings in The American Journal of the Medical Sciences [2], that the patients with non-severe CAP fulfilling the predictive findings most strongly associated to mortality, i.e. $\mathrm{PaO} 2 / \mathrm{FiO} 2 \leq 250 \mathrm{mmHg}$, confusion, and uremia, demonstrated higher SOFA and PSI scores and mortality rates, and might have the priority for treatment and intensive care. Therefore, in Respiratory Research [1], we further proposed a scored minor criteria scoring system which orchestrated improvements in predicting mortality and severity in patients with CAP, and suggested that scored minor criteria of $\geq 2$ scores or the presence of 2 or more IDSA/ATS minor criteria might be more valuable cut-off value for severe CAP.

We apologize for the inappropriate overlap between our three publications and our lack of transparency about the similarities between the three articles.

\section{Author details}

${ }^{1}$ Department of Respiratory Medicine, Shenzhen Hospital, Peking University, Lianhua road No. 1120, Shenzhen 518036, Guangdong, China. ²Department of Respiratory Medicine, The Eighth Affiliated Hospital (Shenzhen Futian), Sun Yat-sen University, Shenzhen 518033, Guangdong, China. ${ }^{3}$ Medical Department, The Eighth Affiliated Hospital (Shenzhen Futian), Sun Yat-sen

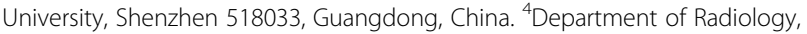
The Eighth Affiliated Hospital (Shenzhen Futian), Sun Yat-sen University, Shenzhen 518033, Guangdong, China. ${ }^{5}$ Guangzhou Institute of Respiratory Diseases (State Key Laboratory of Respiratory Diseases), First Affiliated Hospital, Guangzhou Medical University, Guangzhou 510120, Guangdong, China.

Received: 4 July 2019 Accepted: 4 July 2019

Published online: 10 July 2019

\section{Reference}

1. Guo et al. (2019) Scored minor criteria for severe community-acquired pneumonia predicted better (2019) 20:22 DOl: https://doi.org/10.1186/s12 931-019-0991-4.

\footnotetext{
* Correspondence: qiguo007@sina.com

${ }^{\dagger}$ Qi Guo, Wei-dong Song and Hai-yan Li contributed equally to this work.

${ }^{1}$ Department of Respiratory Medicine, Shenzhen Hospital, Peking University,

Lianhua road No. 1120, Shenzhen 518036, Guangdong, China

${ }^{2}$ Department of Respiratory Medicine, The Eighth Affiliated Hospital

(Shenzhen Futian), Sun Yat-sen University, Shenzhen 518033, Guangdong,

China

Full list of author information is available at the end of the article
}

(c) The Author(s). 2019 Open Access This article is distributed under the terms of the Creative Commons Attribution 4.0 International License (http://creativecommons.org/licenses/by/4.0/), which permits unrestricted use, distribution, and reproduction in any medium, provided you give appropriate credit to the original author(s) and the source, provide a link to the Creative Commons license, and indicate if changes were made. The Creative Commons Public Domain Dedication waiver (http://creativecommons.org/publicdomain/zero/1.0/) applies to the data made available in this article, unless otherwise stated. 